\title{
Caregivers' Supportive Needs when Caring for Chronically-Ill Patients: A Descriptive Qualitative Study
}

\author{
Goh BBL ${ }^{1}$, Wu VX' ${ }^{2}$ AngJ YH' ${ }^{1}$ and Klainin-Yobas \\ $\mathbf{P}^{2 *}$ \\ ${ }^{1}$ Sengkang General Hospital, Singapore \\ ${ }^{2}$ Alice Lee Centre for Nursing Studies, National University \\ of Singapore, Singapore \\ *Correspondling author: Klainin-Yobas P, Alice \\ Lee Centre for Nursing Studies, National University of \\ Singapore, Level 2, Clinical Research Centre, Block MD11, \\ 10 Medical Drive, Singapore
}

Received: August 27, 2021; Accepted: September 30, 2021; Published: October 07, 2021

\begin{abstract}
This study aimed to explore supportive needs of caregivers when caring for patients with chronic illness during hospitalization. A descriptive qualitative study was conducted at a tertiary hospital and purposive sampling was used to recruit 13 participants. Eligibility criteria included primary caregivers of patients with chronic illness. Data were collected via interviews. Results were analyzed using thematic analysis. Five themes were derived, portraying caregivers' perceptions during the patients' hospitalization. The caregivers generally appreciated patient care provided by healthcare professionals. However, they expressed the needs for complete and updated information regarding the patients' conditions and training programs to enhance their knowledge and skills. Furthermore, they requested services to minimize their emotional and financial issues. Addressing caregivers' supportive needs is important to augment the recovery of the patients and to reduce the rate of readmissions. Caregiver support programs can be offered to minimize emotional stress and to enhance their knowledge and skills.
\end{abstract}

Keywords: Chronic illness; Primary caregivers; Supportive needs

\section{Introduction}

Chronic illness signifies conditions with a long-standing duration, lasting three months or more and it is not preventable by vaccines or fully resolved by medications [1]. Such conditions may trigger a change of dependency and dynamics between patients and their caregivers. Moreover, chronic illness may affect the emotional well-being of the family members [2].

Most chronic conditions branch from four main categories; cardiovascular diseases, cancer, chronic respiratory diseases and diabetes mellitus [3]. Almost 70\% of deaths annually are caused by these chronic diseases [4]. In 2014, approximately 133 million or $40 \%$ of Americans were diagnosed with chronic conditions [5]. By 2020 , that number would rise to 157 million with at least $50 \%$ of them experiencing multiple chronic conditions. In Singapore, the prevalence of diabetes, hypertension and hyperlipidemia among adults increased to $4 \%, 14 \%$ and $33 \%$ respectively [6].

The roles of caregivers include assisting with patients' Activities of Daily Living (ADL), psychosocial support, and direct medical or nursing care (such as managing appointments, medication administrations and symptom management) [7]. Among the caregivers, there are formal (paid professionals) and informal types (family or friends) [8]. The informal caregivers are sub-divided into primary and secondary ones. Immediate family members are classified as primary caregivers, while relatives, friends or paid helpers are considered secondary caregivers [9]. In Singapore, shrinking family sizes and higher proportions of older adults make caregivers struggle to maintain their health and work-life balance [10].

Caregivers generally experience a lack of confidence in caring of patient's post-discharge [11]. Being unsupported and uninvolved during the hospitalization period strike caregivers regardless of their level of preparedness [12]. The lack of timely management and lifestyle changes can take a toll on the physical and mental states of caregivers. Disruptions to normal life routines (such as meal and sleep schedules) are common [13]. Fatigue sets in when chronicallyill patients remain dependent for a long time. The problems are more prominent after the patients discharge as the attention and care are no longer split among health professionals. Furthermore, psychological health is an equally significant aspect for caregivers. In a study involving caregivers of cancer patients, those who received caregiver support reported lower risks of depression and anxiety [14]. Therefore, early preparation and support should be initiated to keep psychological stress under control.

In Asia, cultural practices and upbringing generally dictate that family members should adopt the role of caregiver when needed $[15,16]$. Singaporeans generally practice the act of filial piety, which is a value endorsed into the Asian cultures [17]. Given the nature of caregiving practice, it highlights the need to understand caregivers' concerns and experiences, equip them with essential knowledge and skills, and provide supportive needs in a timely fashion.

Supportive needs refer to acquiring medical information or skills through caregiver training, patient education, emotional and psychological support, financial support, assistance with ADLs and access to support groups and facilities $[18,19]$. The term 'needs' can be generalized and non-specific [20]. Existing international and local interventions to support caregivers include: relaxation training, communication skills training, early discharge instructions and training with written information, telephone support hotlines, psychomotor skills, medication knowledge and administration, psycho-education and access to support services [21-25]. Each caregiver's needs are unique, depending on the patient's conditions or external factors affecting the role of the caregiver [26]. Caregivers' needs are dynamic and may change throughout the process of patient care $[21,27,28]$. The capability of the caregivers would affect the 
patients' recovery process and reduce a readmission risk [29].

Previous research explored caregivers 'experiences and needs; however, knowledge gaps still exist. Some studies focused only on one type of chronic condition such as stroke or cancer [27,30]. Such findings may not be transferable to other chronic conditions. Other research examined caregivers' challenges on only one particular time point (such as post-intensive care or post-discharge period) $[11,31]$. Therefore, information at other time points is not wellunderstood [32]. Furthermore, little is known about the supportive needs of caregivers, especially in Singapore. Existing evidence from other countries may not be applicable to Singapore, whose cultural practices, beliefs and healthcare systems are different.

This study aimed to explore caregivers' supportive needs when caring for chronically-ill patients during hospitalization. Findings from this study would help gain in-depth knowledge concerning caregivers' supportive needs and training programs in Singapore. Additionally, the findings would inform clinical practice and healthcare policy on how to better cater to the needs of both caregiver caregivers and patients. These might enhance quality care and minimize patients' risk of readmission.

\section{Material and Methods}

\section{Research design}

A descriptive qualitative design was adopted. This approach allowed the researchers to explore natural perspectives of different individuals to gain insight of certain phenomena [33]. It is one of the qualitative methods, which is more flexible concerning the use of a theoretical framework (i.e. it is not compulsory to use theories to guide the research) [33].

\section{Participants and setting}

This study was conducted at inpatient wards (such as general medicine and cardiology) in a tertiary hospital in Singapore. Participants were recruited via purposive sampling. Selecting caregivers with specific traits are important in ensuring that they contribute to information-rich and relevant data to the research pool [34]. It also helps to minimize influences due to other unrelated factors that may affect findings [35]. Therefore, caregivers were split into four main categories based on patients' chronic conditions: Cancer, Renal, Respiratory and Cardiovascular [3]

Eligibility criteria were: a) primary caregivers of patients who had chronic illness lasting at least three months, b) adults aged 21 to 65 years, and c) able to speak English or Chinese. The primary caregivers were chosen because they actively involved in overall planning and caring for the patients. Paid helpers were excluded because they would passively follow instructions from family members.

According to previous similar qualitative studies, sample sizes ranged from 10 to 44 [12,19,24,26,29,36-38]. For this study, a target sample size of 30 would be used or until data saturation has been achieved (i.e., no new information was added to the research data) [39].

\section{Data collection}

Following the approval of Hospital Ethics Committee, we seek permission from the chief nurse and senior nurse managers to collect data. We screened potential participants using a hospital electronic system and approached those who fulfilled the eligibility criteria. A patient information sheet (PIS) was given to potential caregivers for reference. Sufficient time was given for caregivers to consider participation at their own convenience. Furthermore, it was emphasized that participation was completely voluntary, and no coercion was used. Interested caregivers were brought to a private room, where they were invited to sign a consent form and to complete a socio-demographic sheet.

Data were collected using face-to-face interviews guided by an interview schedule. Interview questions were created based on modifications and references from previous studies $[19,24,27,29,36,37,40]$. Consensus was achieved among the researchers regarding the appropriateness of the questions. Prompting questions were asked if more information was required. The first author interviewed all participants to ensure consistency. The interview sessions lasted from 30 to 45 minutes and each interview was audiorecorded using a digital recorder. Field notes were created to record caregivers' non-verbal behaviors (such as body language and facial expressions), reflecting caregivers' emotions.

\section{Data analysis}

Data collection and data analysis were performed concurrently. Each audio-recorded interview was transcribed verbatim and the transcript was doubled-checked against the original record to ensure accuracy. Participants were then assigned pseudonyms (such as Participant $1=\mathrm{P} 1$ ). The same researcher conducted the interview and transcribed the data to ensure consistency of interview techniques and questioning [41].

Thematic analysis was performed with the following steps: 1) familiarity with the research data, 2) creating initial codes, 3 ) generating themes, 4) refining themes, 5) defining and naming themes, and 6) writing a report [41]. All researchers reviewed and refined the emerging themes/subthemes. Any differing opinions were resolved through discussions until consensus was reached. Data saturation was reached at the $10^{\text {th }}$ caregiver; however, we collected three more caregivers to confirm the saturation.

\section{Ethical considerations}

Ethical approval was obtained from the Hospital Institutional Review Board (Reference number: CIRB2019/2602). Information concerning this research was explained to the participants with emphases on voluntary participation, audio-recoding, confidentiality, and their right to withdraw from the study. All research data were strictly secured in a password-protected computer and can be accessed by only the research team.

\section{Trustworthiness}

Trustworthiness comprises credibility, dependability, transferability, and confirmability [42]. Credibility was ensured by cross-checking the transcripts against the audio-records multiple times to ensure true representation of participants' perception [43]. Member checking with each participant was performed during the interview to ensure correct interpretations of the participant's responses. Dependability was achieved through an audit trail containing research decisions, data collection and data analyses [44]. Records of non-verbal communications in the field notes allowed within-method data triangulation, when comparing the 
notes with their corresponding interview transcripts [45]. The researchers provided the data that made transferability judgements possible for potential users [42]. Specifically, we created a thick description of research process (such as data collection, recording and analysis methods) to ensure that the information can be traced [46]. Additionally, confirmability is established when credibility, transferability and dependability are achieved.

\section{Results}

\section{Participants' socio-demographic data}

Overall, 100 participants were approached but 14 agreed to take part in the study, making a response rate of $14 \%$. Common reasons for the low response rate were disinterest in the research topic or feeling uncomfortable to sit through an audio recording. One interview was discarded due to a dysfunctional audio-recorder. Therefore, the final sample included 13 caregivers and their characteristics are summarized in Table 1. Medical conditions of their patients were summarized in Table 2. An almost equal split of gender was achieved to ensure the balance of opinions from both genders, despite females being the majority of caregivers [47]. Most caregivers aged above 40 years (Mean age $=51.3$ ) and about two-third of the caregivers were employed while being a caregiver. Most of them had no prior caregiving experiences.

\section{Caregivers' supportive needs when caring for chronically- ill patients}

Findings from the thematic analysis suggested five major themes (Table 3-5). All caregivers in this study shared their experiences when their loved ones were hospitalized due to chronic illness. They appreciated all Healthcare Professionals (HCPs) who did a great job in providing care to the patients. However, many informants expressed their needs for more caregiver support. Specifically, they requested for more information support, including clear, jargonfree, straightforward, complete and updated details of the patients' conditions and treatments. Furthermore, many caregivers reported the lack of knowledge and skills concerning patient care. Therefore, they asked for formal and informal trainings to enhance their knowledge and skills before the patients were discharged from hospital. At post-discharge, the caregivers requested a hotline service; and ongoing education sessions and trainings. Additionally, many caregivers, who experienced emotional difficulties and financial challenges, expressed their needs for emotional support, group support and financial advice. Details for each theme are elaborated in the following sections.

Theme 1: Caregivers' perception concerning healthcare professionals and policies: An admission to hospital is recognized as a stressful event for patients and their caregivers. Many respondents in this study verbalized that they needed help and support from Healthcare Professionals (HCPs). They perceived HCPs as those were genuine and dedicated to caring for their patients. Although the caregivers were aware that HCP had busy schedules, they often felt frustrated when they received delayed responses from nurses and doctors. Some respondents felt that HCPs' attentions were given to patients but hardly to caregivers. They also felt that they did not receive sufficient caregiver support (Table 3, Quote 1.1). Furthermore, some opinions revolved around hospital visiting hours and accessibility system (such as the use of access card and long queue), which were
Table 1: Summary of participants' characteristics.

\begin{tabular}{|c|c|c|c|}
\hline \multirow{2}{*}{\multicolumn{2}{|c|}{ Demographic Data }} & \multicolumn{2}{|c|}{ Total $(n=13)$} \\
\hline & & Frequency & Percentage (\%) \\
\hline \multirow{2}{*}{ Gender } & Male & 6 & $46.20 \%$ \\
\hline & Female & 7 & $53.80 \%$ \\
\hline \multirow{4}{*}{ Race } & Chinese & 9 & $69.20 \%$ \\
\hline & Malay & 0 & - \\
\hline & Indian & 4 & $30.80 \%$ \\
\hline & Others & 0 & - \\
\hline \multirow{5}{*}{ Religion } & Christianity & 1 & $7.70 \%$ \\
\hline & Buddhism & 4 & $30.80 \%$ \\
\hline & Muslim & 0 & - \\
\hline & Hinduism & 2 & $15.40 \%$ \\
\hline & Others & 6 & $46.20 \%$ \\
\hline \multirow{4}{*}{ Age } & $18-30$ & 1 & $7.70 \%$ \\
\hline & $31-40$ & 1 & $7.70 \%$ \\
\hline & $41-50$ & 4 & $30.80 \%$ \\
\hline & $51-65$ & 7 & $53.80 \%$ \\
\hline \multirow{5}{*}{ Education Level } & Primary School & 0 & - \\
\hline & Secondary School & 5 & $38.40 \%$ \\
\hline & Diploma/Nitec & 4 & $30.80 \%$ \\
\hline & Degree \& above & 4 & $30.80 \%$ \\
\hline & None & 0 & - \\
\hline \multirow{4}{*}{ Relationship with patient } & Children & 9 & $69.20 \%$ \\
\hline & Spouse & 4 & $30.80 \%$ \\
\hline & Sibling & 0 & - \\
\hline & Relative & 0 & - \\
\hline \multirow{4}{*}{ Marital Status } & Single & 1 & $7.70 \%$ \\
\hline & Married & 11 & $84.60 \%$ \\
\hline & Divorced & 1 & $7.70 \%$ \\
\hline & Widowed & 0 & - \\
\hline \multirow{2}{*}{ Working Status } & Working & 9 & $69.20 \%$ \\
\hline & Not working & 4 & $30.80 \%$ \\
\hline \multirow{3}{*}{$\begin{array}{l}\text { Length of caring } \\
\text { experience }\end{array}$} & $<1$ month & 3 & $23.10 \%$ \\
\hline & 1-6 months & 2 & $15.40 \%$ \\
\hline & $>6$ months & 8 & $61.50 \%$ \\
\hline \multirow{2}{*}{ Previous caring experience } & Yes & 3 & $23.10 \%$ \\
\hline & No & 10 & $76.90 \%$ \\
\hline
\end{tabular}

inconvenient. Some caregivers requested for more flexible visiting hours that would accommodate their work and daily life schedules. Concerning a need to obtain information updates, some caregivers had a hard time to meet doctors due to conflicts between the visiting hours and doctors' rounds (Table 3, Quote 1.2).

Theme 2: Acquiring and managing patient-related information: Information concerning the patients' health conditions and regular updates were crucial for the caregivers. Such information could have minimized their anxiety and nervousness. Most HCPs provided the 
Table 2: Categories of patients' medical conditions.

\begin{tabular}{|c|c|c|c|c|}
\hline Patient & Cardiovascular & Respiratory & Cancer & Renal \\
\hline P1 & & $\checkmark$ & $\checkmark$ & \\
\hline P2 & $\checkmark$ & & & $\checkmark$ \\
\hline P3 & $\checkmark$ & & & \\
\hline P4 & $\checkmark$ & $\checkmark$ & & \\
\hline P5 & & & $\checkmark$ & \\
\hline P6 & $\checkmark$ & & $\checkmark$ & $\checkmark$ \\
\hline P7 & & $\checkmark$ & & $\checkmark$ \\
\hline P8 & & $\checkmark$ & & \\
\hline P9 & $\checkmark$ & $\checkmark$ & & $\checkmark$ \\
\hline P10 & & & & $\checkmark$ \\
\hline P11 & & & & \\
\hline P12 & $\checkmark$ & & & \\
\hline P13 & $\checkmark$ & & & \\
\hline
\end{tabular}

information to the caregivers on a regular basis. Nevertheless, some participants expressed that they received unclear and incomplete medical information. They suggested that HCPs should have offered the patient's information even without asking. Some caregivers learned about the patients' medical diagnosis; however, they did not know the meaning of such medical terms. Therefore, they resorted to internet to solve the unmet information needs (Table 3, Quote 2.1) Furthermore, some caregivers felt that they did not receive constantly updated information regarding interim diagnosis, test results and patients' conditions. Other caregivers received mismatched information resulting in confusion. Contradictory information sometimes occurred when they were updated by two different doctors (Table 3, Quote 2.2). Consequently, it was preferable that the relay of information was conveyed by only one HCP (Table 3, Quote 2.3)
Theme 3: Empowering with knowledge and skills: During the patents' hospitalization, all informants became aware that they needed to engage in the patient care to some extent. Some of them would stay overnight to accompany the patients and to provide help with ADLs. Nonetheless, more than half of the caregivers did not feel confident in caring for the patient during hospitalization (Table 4, Quote 3.1). They perceived the lack knowledge and essential skills. Therefore, they felt worried, especially during the overnight stays when fewer HCPs working on evening and night shifts. Fortunately, some caregivers received informal trainings in the study hospital on certain skills such as assisting with exercise or medical procedures. Despite that, many participants requested for more formal education and training sessions to enhance their caregiving knowledge and skills. Additionally, one participant suggested that monthly education sessions be offered to caregivers to provide ongoing knowledge and to clarify issues that they might have (Table 4, Quote 3.2).

Theme 4: Overcoming challenges in caregiving: Taking care of chronically-ill patients are challenging to all caregivers. Many respondents expressed their emotional problems such as feelings of uncertainty, nervousness, anxiety, fear, and stress. The intensity of emotional stress depended on the patients' conditions. Issues that triggered the uncertainty included determining the disease progression, recovery of the patients' conditions, financial capability, or follow-up treatments. Financial burden was a main contributor to emotional stress. Some respondents reported their income loss after taking leave of absence from work to accompany the patients. Furthermore, medical costs required long-term planning. Most caregivers struggled to pay the medical bills even though they received some governmental subsidy. Sometimes, they had to use their own savings to alleviate the financial burden (Table 4, Quote 4.1).

To manage stress, some caregivers preferred self-management strategies such as reading spiritual books or listening to radio.

Table 3: Participants 'quotes for theme 1 and 2

Themes

Caregivers' perception concerning healthcare professionals and policies

\section{Participants' quotes}

1.1 Experiences with healthcare professionals

"The healthcare professionals are dedicated people. They genuinely care for the patients and family members but at time maybe there could be blind spots. I was also touched by the ward staff, the way that they interact my (with) mum. I can feel that they are genuine."[P4]/17, 21.

"You will need help but sometimes the staff are very busy so we have to just wait. Sometime the wait is a bit long. It is also frustrating like we try and get help here but then it's like..."[P1]/47.

1.2 Perception and issues towards hospital visiting hours policy

"Reception downstairs they will tell you that we will let you go in this time round but please visit during the visiting hours. We do understand the need for patient to rest. It's not that we wanted to disturb, but then... The patient sometimes maybe they couldn't sleep or what, they really want to see their family members."[P1]/16.

"We have visitors during the visiting hours but they were denied to come in, even though we are not at cap of four persons. You exit, this person left, is still in the record. Then what is the purpose having such gantry? This person left, you have three still in the ward, and that one more person can actually visit her. We have to go to the counter, queue up again" [P7]/42, 44, 46, 50. 2.1 Enquiring early and regular updates of patient-related information

"They might not give you the full information, even you asked. They will give you a partial. Then they will check and get back to you, or some don't even bother to ask..."[P7]/17.

"...When you see the caregiver, the related (HCP) should volunteer instead of having the person sitting there and guessing. We have to keep probing. You hoped for like auto update kind of process" [P11]/204, 206, 209

2.2 Barriers receiving patient health-related information

"This doctor was telling me about the kind of the operation and later on another doctor who came and tell me...might be going 2. Acquiring and managing patient- through is totally different. I am receiving two sub-information from two different doctors so what is what?"[P1]/57, 60,64 . related information "I think they are quite consistent the nurses are very clear in terms of knowing that if there are some questions that only the doctor can answer. The nurse doesn't try to do a doctor's job so when that happens, so most of the time you kind of get answers that...In some sense, it's consistent that way I would say."[P12]/105, 107.

2.3 Information needs from healthcare professionals

"I do hope that I can have just one doctor, who provide me with the very confirmed information on the next step we are going to do. You give me one definite answer and not different type of things from different doctors."[P1]/249

"...rather than to communicate from a medical stand point. So, being able to very easily break down the big terms into what it means for layman."[P12]/442, 444 
Table 4: Participants' quotes for theme 3 and 4. Themes

Participants' quotes

\begin{tabular}{|c|c|}
\hline Themes & Participants' quotes \\
\hline $\begin{array}{l}\text { 3. Empowering with } \\
\text { knowledge and skills }\end{array}$ & $\begin{array}{l}3.1 \text { Lack of knowledge and skills } \\
\text { "The nurses...they will come by "Who is staying overnight?" but it also gives impression. Are we on duty to take (care) of her? If we } \\
\text { really could not stay overnight, what we going to be expected to have death in this hospital provided to the patient? That give me the } \\
\text { impression that...a bit of responsibility is given to us but not knowing what to do."[P7]/83. } \\
\text { "Let's say go back...like the need to help the patient. Then, I think about the training or what by the hospital staff, okay. They still did } \\
\text { teach...like how to pull up the patient, all these things. Or then some of the light things, exercises, or what to do."[P5]/57, } 63 \text {. } \\
3.2 \text { Request for regular training sessions } \\
\text { "Ya of course they must include caregivers...I think they haven't come to the extent of that...If not how would the family member even } \\
\text { care for the elderly"[P13]/115, 117, 133. } \\
\text { "Maybe can have some short talk organized by the hospital. Maybe once a month...like by a doctor whom you can ask questions. It's } \\
\text { a good chance at least we can ask. Or maybe it can be conducted by a nurse or whoever have more knowledge in the field."[P1]/106, } \\
108 .\end{array}$ \\
\hline $\begin{array}{l}\text { 4. Overcoming emotional } \\
\text { challenges in caregiving }\end{array}$ & $\begin{array}{l}\text { 4.1 Emotional issues and uncertainties faced in patient care } \\
\text { "Some caregivers, they are not working so there is no income for them. So financial wise, they definitely need support. The caregiver's } \\
\text { needs is the mental and the financial support. That's very important for caregiver." [P5]/101,236,238 } \\
\text { "There's always a fear of losing somebody close... maybe every time she comes in. And my mum being eighty years old and she is } \\
\text { also having this concurrent heart problem, which is like a living time bomb with you. So...maybe more on emotional fear every time she } \\
\text { comes in here. Everybody pushes the panic button...so everybody drops everything and comes." [P3]/87, } 92 . \\
4.2 \text { Self-management of emotional issues } \\
\text { "I guess it's good for me because I can rotate among a few people. But, I can imagine how if you are doing it alone, then it is super } \\
\text { heavy..."[P12]/18. } \\
\text { "I think sometimes you get a bit stressful when she (patient) being emotional. Sometimes, speak certain words that are hurtful. We just } \\
\text { ignore. Try our best to. Only occasionally. It's like take it as something is part of the sickness. You just got to tolerate a bit more. Of } \\
\text { course, if I flare up, she flare up, then there is no end"[P6]/166. } \\
4.3 \text { Needs for emotional support } \\
\text { "A lot of, a bit more care and concern should be provided to the family members. To speak to them more. Communication is the best" } \\
\text { [P13]/267. } \\
\text { "I would say that there is people who are helpful here. That's really touching. It's something that we didn't request but is given the extra } \\
\text { miles. All these are extra miles that it really makes a caregiver or anyone here feel the touch."[P7]/137 } \\
\text { "I got my own things to do. I don't even get enough sleep. I want to contact them. It takes time for me to...but I do not know. I really am } \\
\text { not very familiar with it. I've never went...online to see whether there is such...a group and how whether it's pertaining to your needs or } \\
\text { not...your illness..."[P11]/124. }\end{array}$ \\
\hline
\end{tabular}

Table 5: Participants 'quotes for theme 5. Themes Participants' quotes

5.1 Discharge preparation received from healthcare professionals "Normally they will come around and then they will give you a form and then said go and get all these medications, then said come back as outpatient, and give you appointments..."[P11]/137.

5.2 Readiness for homecare

"Upon discharge, the patients will mostly be dependent on the caregivers. If during the stay in the hospital, the family members do not hear from the doctor and the staff nurse are also very careful about what they inform the caregiver, how does the hospital expect the caregiver to be very confident? If there is no communication, which is a missing thing..."[P4]/112, 117.

"It's a good initiative that if the hospital will be able to train a caregiver if we are bringing her home. How should we take care of her? We

5. Transitioning from are anxious to know. Even I was just now asking the two nurses doing it...where can I help out...to learn. Then they said, it's ok they can manage."[P7]/143, 145.

hospital to homecare

"Even the patient is not fully recovered or what. I think they also want them to discharge because they are viruses in the hospital or what. I will... a bit disagree...other of my visitor I talked too they also have this issue."[P5]/165, 171.

5.3 Needs for helpline services post-discharge

"Once you get out of hospital, there is no way you can call anybody. That is one big problem...There is no such thing as common reception...But if I could call someone, then it could help...But, unfortunately, there isn't such a things as general helpline for medical or caregiver whatever you call it."[P6]/147, 268.

"...such a helpline will not be very helpful because people won't want to commit one way or the other you know...I will say better be safe than sorry...Because if you really want to give a proper answer to that question then somebody got to come and check out the patient...?"[P8]/127.

Among family members, they took turns taking care of the patients to minimize stress levels. Other caregivers felt that it would help if they could seek assistance from another caregiver (Table 4, Quote 4.2). Furthermore, most participants had positive experiences and received great help from the HCPs. However, others hoped that the HCPs could have provided more emotional support or assist in financial aids to those who had difficulties. Additionally, attending support groups was perceived as an option to help with individuals' needs. However, one of the most common obstacles was a lack of time. Not all caregivers were inclined to attend the support groups. They were not interested or felt that it would not be as useful as family support (Table 4, Quote 4.3).
Theme 5: Transitioning from hospital to homecare: Knowing that the chronically-ill patients were discharged from hospital was supposed to be good news for all caregivers. Nonetheless, some caregivers expressed their concerns that the patients were discharged without a full recovery. Although the study hospital provided discharge preparation, some caregivers still felt incompetent and unprepared. They reported the lack of physical ability, knowledge, or experience as they were informed not long before the discharge date. Some caregivers felt that HCPs did not take into consideration the caregivers' readiness before discharging the patients. They hoped that HCPs would have informed them discharge date in advance, provided essential knowledge and taught skills earlier so that they 
could practice the skills under HCPs' supervision (Table 5, Quote 5.1, 5.2). Furthermore, most caregivers requested a helpline service provided by HCPs at post-discharge to help them clarify issues such as decision making on patient care or medications (Table 5, Quote $5.3)$.

\section{Discussion}

This study aimed to explore the supportive needs of primary caregivers of chronically-ill patients during hospitalization. Findings included five major themes. The first two themes focused on the caregivers' perception on HCPs and their needs for information support including clear, jargon-free, straightforward, complete and updated details of the patients' conditions and treatments. However, they did not obtain the formation due to HCPs' busy schedules and the visiting hours that did not allow an opportunity to meet doctors during ward rounds. Similarly, a previous study documented that caregivers of cancer patients faced difficulties obtaining information from HCPs especially from nurses and doctors [48]. Therefore, the study suggested that HCPs should communicate effectively with patients and caregivers throughout the hospitalization period. HCPs should not assume that caregivers are responsible to search internet to enhance their knowledge. Essential information (such as medical diagnosis, assessments, treatments, and resources) should be provided clearly via verbal, written and electronic platforms.48 Furthermore, HCPs should simplify medical jargons and should not just deliver hurling chains of medical terminologies [28].

The visiting hour policy contributed to frustrations among the caregivers. In the study hospital, visiting hours included two slots: $12: 00 \mathrm{pm}-2: 00 \mathrm{pm}$ and 5:00pm-8:00pm. Furthermore, up to four visitors were allowed per patient. The visitors had to register every time they enter or re-enter a building entrance even on the same day. They sometimes faced a long queue during the registration. Therefore, many caregivers expressed the needs for a more convenient registration system and more flexible visiting hours. A previous research reported that open visiting hours were more beneficial. Flexible hours allowed caregivers with various working schedules to visit patients according to their schedules [49]. Although the visiting policy is meant to minimize disruptions to HCPs' routine work and to allow patients to rest, there is little evidence to support that notions [50]. In fact, the presence of family members may be emotionally beneficial and provide essential support to the patients [51].

Although nurses in the study hospital provided quality care to the patients, most caregivers had to assist with patients' ADLs to a certain degree. They stated that they had insufficient knowledge and skills to provide care to the patients during hospitalization and postdischarge. Consequently, they expressed the needs for trainings and discharge preparation to enhance knowledge and skills. A previous study found that caregivers who were enrolled in structure training sessions during hospitalization reported increased self-efficacy, preparedness and psychological well-being [52]. Furthermore, a good discharge preparation process should begin at the start of hospitalization. A proper guidance for caregivers would ensure that they are more confident to manage the patients alone following discharge [53]. Additionally, the lack of caregivers' readiness upon discharge was partly associated with mismatched expectations in patients' condition for discharge. Therefore, active communications between HCP and caregivers would reduce the misunderstanding and mismatched expectations [54].

More than half of the caregivers in this study expressed financial concerns and emotional problems, including stress, anxiety and fear. They expressed the needs for emotional support provided by HCPs during hospitalization; and helpline services, support groups and regular education sessions post-discharge. A previous study reported that Singaporean caregivers had lower quality of life in comparison with Western counterparts [55]. However, support groups may be beneficial for caregivers. An experimental research reported that a 12-week support group significantly reduced depression and burden among dementia caregivers in Taiwan [56]. Support groups serve as a platform for caregivers to exchange information in an emotionally supportive environment and to provide social support for group members. Other interventions to support caregivers include relaxation training, communication skills training, early discharge instructions, psychomotor skills, medication knowledge and administration, psycho-education and access to support services [21-25].

\section{Strengths and Limitations of This Study}

This study used a qualitative design, an ideal approach for eliciting in-depth information concerning the caregivers' perceptions. The maximum variation sampling allowed the recruitment of caregivers from different chronic conditions, reflecting the diversity of participants. However, there were some methodological limitations. The caregivers were selected from only one tertiary hospital in Singapore and the response rate was low (14\%). Such issues might limit the transferability of findings. Furthermore, Singapore comprises three major ethic groups (Chinese, Indian and Malay) but only Chinese and Indian caregivers were interviewed. This issue was restricted by the researcher's limited language proficiency. Future research may explore cultural differences among racial groups using different languages.

\section{Implications for Clinical Practice}

Findings from this study highlighted the caregivers' needs for; complete and updated patient-related information, education and training sessions and emotional support programs. The HCPs performed well in providing quality care to the patients. However, they may need to communicate more with the caregivers and to update patients' information daily. A caregiver support center may be established and serve as a resource center to provide patients' information, medical knowledge, and skills training for the caregivers. Several training programs can be offered to teach caregivers to assist with patients' ADLs (such as showering, toileting, exercising and wound care). Furthermore, HCPs may refer the caregivers to existing resources such as helpline services, caregiver network, and caregiver support groups. A list of relevant numbers and/or websites of available services should be provided. The helpline services should provide jargon-free information regarding medical diagnosis and treatments. The caregiver network and support groups may serve as platforms for caregivers to exchange information, share experiences and provide emotional support for each other.

\section{Recommendations for Future Research}

Future research may explore caregivers' supportive needs using 
multiple assessment points such as during hospitalization, transitional care and home settings. A mixed method may be utilized with a larger sample size and multi-center settings to be representative of caregivers. A quantitative approach using self-reported questionnaires with multiple languages (such as Chinese, Indian, Malay and English) may be conducted. Using the questionnaires instead of audio-recorded interview may be more socially comfortable for those who are more reserved. Given that some issues emerged during the interview (such as manpower shortage), future studies may explore challenges that HCP face in meeting the demands of caregivers. Additionally, experimental research may be carried out the evaluate effectiveness of the caregiver support programs, training programs and support groups.

\section{Conclusion}

This study aimed to explore the supportive needs of primary caregivers of chronically-ill patients during hospitalization. Thematic analysis revealed five major themes, including caregivers' perception concerning healthcare professionals and policies, acquiring and managing patient-related information, empowering with knowledge and skills, overcoming emotional challenges in caregiving, and transitioning from hospital to homecare. Findings in this study may inform clinical practice whereby a caregiver support center and caregiver training programs may be established. Future research may test the effectiveness of the training programs on physical and emotional health among caregivers of chronically-ill patients.

\section{References}

1. Bernell S \& Howard SW. Use Your Words Carefully: What Is a Chronic Disease? Front Public Health. 2016; 4: 2-4.

2. Ehsan N, Johar N, Saleem T, Khan MA \& Ghauri S. Negative repercussions of caregiving burden: Poor psychological well-being and depression. Pak J Medical Sciences. 2018; 34: 1452-1456.

3. World Health Organization. Global status report on noncommunicable diseases. 2014.

4. McGrady A \& Moss D. Integrative pathways: Navigating chronic illness with a mind-body-spriti approach. New York, NY: Springer. 2018.

5. Agency for Healthcare Research and Quality. Multiple Chronic Conditions Chartbook: 2010 Medical Expenditure Panel Survey Data. 2014.

6. Minister of Health, Singapore. The Burden of Disease in Singapore, 1990 2017: An overview of the Global Burden of Disease Study 2017 results. 2019

7. Mollica MA, Litzelman K, Rowland JH \& Kent EE. The role of medical/nursing skills training in caregiver confidence and burden: A CanCORS study. Cancer. 2017; 123: 4481-4487.

8. Shiba K, Kondo N, \& Kondo K. Informal and formal social support and caregiver burden: The AGES caregiver survey. J Epidemiol. 2016; 26: 622 628.

9. Barbosa A, Figueiredo D, Sousa L \& Demain S. Coping with the caregiving role: Differences between primary and secondary caregivers of dependent elderly people. Aging and Ment Health. 2011; 15: 490-499.

10. Mehta KK \& Leng TL. Experiences of Formal and Informal Caregivers of Older Persons in Singapore. J Cross-Cult Gerontol. 2017; 32: 373-385.

11. Ewing G, Austin L, Jones D \& Grande G. Who cares for the carers at hospital discharge at the end of life? A qualitative study of current practice in discharge planning and the potential value of using The Carer Support Needs Assessment Tool (CSNAT) Approach. Palliat Med. 2018; 32: 939-949.

12. Ang WHD, Lang SP, Ang E \& Lopez V. Transition journey from hospital to home in patients with cancer and their caregivers: a qualitative study. Support
Care Cancer. 2016; 24: 4319-4326

13. Popli UK \& Panday R. Health Problems of Caregivers of Hospitalized Elderly. J Psychosoc Res. 2017; 12: 355.

14. Karabekiroğlu A, Demir EY, Aker S, Kocamanoğlu B, Karabulut GS. Predictors of depression and anxiety among caregivers of hospitalised advanced cancer patients. Singapore Med J. 2018; 59: 572-577.

15. Holroyd EE. Chinese family obligations toward chronically ill elderly members: Comparing caregivers in Beijing and Hong Kong. Qual Health Res. 2003; 13: 302-318.

16. Lee R. Caring for family members with stroke: Chinese family caregivers' experiences. Asian J Nurs studies. 2004; 7: 18-27.

17. Ng HY, Griva K, Lim HA, Mahendran R. The burden of filial piety : A qualitative study on caregiving motivations amongst family caregivers of patients with cancer in Singapore. Psychol Health. 2016; 31: 1293-1310.

18. Au A, Lam W, Tsang J, Yau TK, Soong I, Yeo W, et al. Supportive care needs in Hong Kong Chinese women confronting advanced breast cancer. Psychooncology. 2013; 22: 1144-1151.

19. Lavallée JF, Grogan S, Austin CA. Cancer patients' family members' experiences of the information and support provided by healthcare professionals. Health Educ J. 2019; 78: 416-427.

20. Asadi-Lari M, Tamburini M \& Gray D. Patients' needs, satisfaction, and health related quality of life: Towards a comprehensive model. Health and Qual Life Outcomes. 2004; 2: 1-15.

21. Anjum W. Schizophrenia and Epilepsy; Effectiveness of Relaxation Training for Caregivers of Patients. Prof Med J. 2017; 24: 757-766.

22. Coleman EA, Smith JD, Frank JC, PH SJM, Parry C \& Kramer AM. Preparing Patients and Caregivers to Participate in Care Delivered Across Settings: The Care Transitions Intervention. Circulation. 2015; 9: 200-206.

23. Eggenberger E, Heimerl K \& Bennett MI. Communication skills training in dementia care: A systematic review of effectiveness, training content, and didactic methods in different care settings. Int. Psychogeriatr. 2013; 25: 345 358.

24. Hahn-Goldberg S, Jeffs L, Troup A, Kubba R \& Okrainec K. "We are doing it together": The integral role of caregivers in a patients' transition home from the medicine unit. PLoS ONE. 2018; 13: 1-14.

25. Philip RR, Venables E, Manima A, Tripathy JP, Philip S, Eggenberger E, et al. "Small small interventions, big big roles"- a qualitative study of patient, caregiver and health-care worker experiences of a palliative care programme in Kerala, India. Int Psychogeriatr. 2019; 18: 1-11.

26. Greenwood N, Pound C, Brearley S \& Smith R. A qualitative study of olde informal carers' experiences and perceptions of their caring role. Maturitas. 2019; 124: 1-7.

27. Chen L, Xiao LD \& De Bellis A. First-time stroke survivors and caregivers' perceptions of being engaged in rehabilitation. J Adv Nurs. 2016; 72: 73-84.

28. Chen $\mathrm{KL}$, Chang $\mathrm{CM}$, Chen $\mathrm{CH}$ \& Huang MC. Information Reception and Expectations among Hospitalized Elderly Patients in Taiwan: A Pilot Study. J Nurs Res. 2018; 26: 199-206.

29. White CL, Brady TL, Saucedo LL, Motz D, Sharp J \& Birnbaum LA. Towards a better understanding of readmissions after stroke: Partnering with stroke survivors and caregivers. J Clin Nurs. 2015; 24: 1091-1100.

30. Hashemi-ghasemabadi M, Taleghani F \& Yousefy A. Transition to the new role of caregiving for families of patients with breast cancer : a qualitative descriptive exploratory study. 2016; 1269-1276

31. De Miranda S, Pochard F, Chaize M, Megarbane B, Cuvelier A, Bele N, et al. Postintensive care unit psychological burden in patients with chronic obstructive pulmonary disease and informal caregivers: A multicenter study. Crit Care Med Critical Care Medicine. 2011; 39: 112-118.

32. Cameron JI \& Gignac MAM. "Timing It Right": A conceptual framework for addressing the support needs of family caregivers to stroke survivors from the hospital to the home. Patient Educ and Couns. 2008; 70: 305-314. 
33. Kim H, Sefcik JS \& Bradway C. Characteristics of Qualitative Descriptive Studies: A Systematic Review. Res Nurs Health. 2017; 40: 23-42.

34. Etikan I, Musa SA \& Alkassim RS. Comparison of Convenience Sampling and Purposive Sampling Comparison of Convenience Sampling and Purposive Sampling. American J Theoretical and Applied Stat. 2016; 5: 1-5.

35. Palinkas LA, Horwitz SM, Green CA, Wisdom JP, Duan N, Hoagwood, K. Purposeful sampling for qualitative data collection and analysis in mixed method implementation research. Adm Policy Ment Health. 2015; 42: 533 544.

36. Leow $\mathrm{MOH}$ \& Chan SWC. The challenges, emotions, coping, and gains of family caregivers caring for patients with advanced cancer in Singapore: A qualitative study. Cancer Nurs. 2017; 40: 22-30.

37. Miller AD, Mishra SR, Jendal L, Haldar S, Pollack AH, Pratt W, et al. Partners in care: Design considerations for caregivers and patients during a hospital stay. CSCW Conf Comput Support Coop Work. 2016; 2016: 756-769.

38. Vaingankar JA, Subramaniam M, Picco L, Eng GK, Shafie S, Sambasivam $\mathrm{R}$, et al. A Perceived unmet needs of informal caregivers of people with dementia in Singapore. Int Psychogeriatr. 2013; 25: 1605-1619.

39. Austin Z \& Sutton J. Qualitative research: Getting started. Can J Hosp Pharm. 2014; 67: 436-440.

40. Chan EY, Phang KN, Glass GF \& Lim WS. Crossing, Trudging and Settling: A phenomenological inquiry into lived experience of Asian family caregivers of older persons with dementia. Geriatr Nurs. 2019; 40: 502-509.

41. Braun $\mathrm{V}$ \& Clarke V. Using thematic analysis in psychology. Qua Res Psychol. 2006; 33: 1-41.

42. Lincoln YS \& Guba EG. Naturalistic Inquiry. Newbury Park, CA: Sage. 1985.

43. Carcary M. The research audit trial - enhancing trustworthiness in qualitative inquiry. Electron J Bus Res Methods. 2009; 7: 11-24.

44. Thomas E \& Magilvy JK. Qualitative Rigor or Research Validity in Qualitative Research. J Spec Pediatr Nurs. 2011; 16: 151-155

45. Halcomb EJ, Gholizadeh L, DiGiacomo M, Phillips J \& Davidson PM Literature review: Considerations in undertaking focus group research with culturally and linguistically diverse groups. J Clin Nurs. 2007; 16: 1000-1011.
46. Anney VN. Ensuring the quality of the findings of qualitative research: looking at trustworthiness criteria. J Emerg Trends in Educ Res Policy Studies. 2014; 5: $272-281$

47. Sharma N, Chakrabarti S \& Grover S. Gender differences in caregiving among family-caregivers of people with mental illnesses. World J Psychiatry. 2016; $6: 7$.

48. Reinhard SC, Given B, Petlick NH \& Bemis A. Patient Safety and Quality: An Evidence-Based Handbook for Nurses. 2008.

49. Griffith DNW. Hospital visiting hours. Brit Med J. 1988; 296: 1798-1799.

50. Shulkin D, Keefe TO, Visconi D, Robinson A, Rooke AS \& Neigher W. Eliminating Visiting Hour Restrictions in Hospitals. 2013; 36: 54-57.

51. Hurst H, Griffiths J, Hunt C \& Martinez E. A realist evaluation of the implementation of open visiting in an acute care setting for older people. BMC Health Serv Res. 2019; 19: 867.

52. Hendrix CC, Bailey Jr DE, Steinhauser KE, Olsen MK, Stechuchak KM Lowman SG, ET AL. Effects of enhanced caregiver training program on cancer caregiver's self-efficacy, preparedness, and psychological well-being. Physiol Behav. 2017; 176: 139-148.

53. Bull MJ, \& Roberts J. Components of a proper hospital discharge for elders J. Adv Nurs. 2001; 35: 571-581.

54. Bauer M, Fitzgerald L, Haesler E \& Manfrin M. Hospital discharge planning for frail older people and their family. Are we delivering best practice? A review of the evidence. J Clin Nurs. 2009; 18: 2539-2546.

55. Lim HA, Tan JYS, Chua J, Yoong RKL, Lim SE, Kua EH, et al. Quality of life of family caregivers of cancer patients in Singapore and globally. Singapore Medical J. 2017; 58: 258-261.

56. Chu H, Yang CY, Liao YH, Chang LI, Chen $\mathrm{CH}$, Lin CC, et al. The effects of a support group on dementia caregivers' burden and depression. J Aging Health. 2011; 23: 228-241. 